\title{
Histological Evaluation of Decellularized Skeletal Muscle Tissue Using Two Different Decellularization Agents
}

\author{
Hana Hrebíková*, Magda Voborníková, Milada Hetešová, Jaroslav Mokrý
}

Department of Histology and Embryology, Charles University, Medical Faculty, Hradec Králové, Czech Republic

* Corresponding author: Department of Histology and Embryology, Charles University, Medical Faculty, Šimkova 870 , 50003 Hradec Králové, Czech Republic; e-mail: hrebikh@1fhk.cuni.cz

Summary: The aim of the present study was to determine effect of two decellularized agents, sodium dodecyl sulphate (SDS) and Triton X-100, to the skeletal muscle tissue. Final scaffold was evaluated by several histological techniques to analyse preservation of essential structures including collagen and elastic fibres, basement membranes, glycosaminoglycans and also to confirm elimination of nuclear and cytoplasmic components which are redundant in effectively prepared decellularized scaffolds. Comparison of tissue scaffolds processed with different detergents proved that SDS is superior to Triton X-100 as it can effectively decellularize muscle tissue.

Keywords: Decellularized scaffold; Histology; Skeletal muscle; SDS; Triton X-100

\section{Introduction}

A tissue loss in soft tissues caused by a trauma or a tumour requires a surgical reconstruction. Effective treatment of such defects can be prepared by tissue transplantation. Tissue engineering and regenerative medicine undergo significant development in the area of biomaterials and combination with stem cells offers accessible source for tissue reconstruction and transplantation (1-4). Biological cell-free material can serve as an optimal scaffold for wide applications as it does not evoke an immune response. Extracellular matrix $(\mathrm{ECM})$ is a promising biomaterial alternative which provides a three-dimensional microenvironment niche for cells, which is highly specific for each type of tissue concerning the protein contents and complexity. There are several options how to substitute stromal components for scaffold production using: synthetic scaffold material, chemically cross-linked forms of ECM, purified ECM components or decellularization techniques. Successful decellularization produces biological material which combines advantages and properties of extracellular matrix as supportive microarchitecture, vascular network and participate in cell differentiation and proliferation $(5,6)$. Biological scaffolds composed of the extracellular matrix are commonly used in reconstructive surgical applications of many organs such as urinary bladder $(7,8)$, heart $(9-11)$, lung $(12,13)$ or skin $(14,15)$.

This study compares decellularization effectiveness of two detergents, SDS (sodium dodecyl sulphate) and Triton $\mathrm{X}-100$, which are both widely used as decellularization agents $(16,17)$. These agents supposed to maintain me- chanical and structural integrity of the extracellular matrix with minimal damage of the scaffold, preserve vascular and neural networks and successfully remove nuclear and cytoplasmic components from ECM.

\section{Material and Methods}

All procedures were approved by the Ethical Committee supervising procedures on experimental animals at Charles University Medical Faculty in Hradec Králové.

\section{Dissection of murine skeletal muscle tissue}

A skeletal muscle tissue (approx. $1 \times 0.5 \times 0.5 \mathrm{~cm}$ pieces) was dissected from $\mathrm{C} 57 \mathrm{BL} / 6$ mice and was immediately washed with PBS buffer and trimmed of a connective tissue and fat. The samples $(n=10)$ were fixed and processed for histological analysis.

\section{Decellularization methods}

We analysed two types of decellularization agents: $1 \%$ SDS (sodium dodecyl sulphate) and 1\% Triton X-100 and samples were decellularized with the following protocol. Solutions were dissolved in distilled water.

A tissue was removed under aseptic conditions. Native samples were immediately processed after samples were delivered to the lab. The skeletal muscle was placed in $1 \%$ SDS or $1 \%$ Triton X-100 solution for 24 hours at room temperature under continuous shaking to remove cell components from the muscle tissue. The muscle sheets were 
thoroughly washed with the fresh PBS buffer for $24 \mathrm{~h}$ at $4{ }^{\circ} \mathrm{C}$ to wash out cells and potential SDS or Triton X-100 residues. The samples were used on the same day when they were prepared. All steps were processed in centrifuge tube with approx. $40 \mathrm{ml}$ of solution.

\section{Histological analysis}

The specimens (untreated and decellularized scaffolds) were fixed in $10 \%$ formalin, embedded in paraffin and sectioned into $5 \mu \mathrm{m}$ slices. Sections were deparaffinised, rehydrated and washed with distilled water. For histological analysis, the sections were stained with haematoxylin eosin, Sirius red, Alcian blue, lamina externa was impregnated by Jones' method, and elastic fibres were visualized with resorcin-fuchsin staining.

\section{Results}

\section{Morphological characterization of physiological skeletal muscle}

Native muscle samples were treated with two different decellularization agents and muscle morphology was examined with haematoxylin eosin staining compared to the untreated skeletal muscle sample. Figure 1A shows typical image of skeletal muscle represented by characteristic striated muscle fibres with nuclei localized beneath the sarcolemma revealing polygonal shape in a cross section.

\section{Morphology of decellularized skeletal muscle tissue treated with SDS or Triton X-100 Haematoxylin eosin staining}

SDS decellularization method resulted in a complete cell removal from the skeletal muscle tissue which was verified with standard haematoxylin-eosin staining. Tissue also revealed a general shrinkage. Microscopic architecture of the skeletal muscle tissue was preserved and typical polygonal shape of extracellular matrix, which surrounds empty spaces of striated skeletal fibres, was examined (Fig. 1B). Haematoxylin eosin staining revealed a complete removal of sarcoplasmic components which were washed out with PBS buffer. Three-dimensional architecture was preserved without nuclei remnants supported with DAPI staining showing no fluorescent signal (figure not shown).

Native skeletal muscle tissue treated with SDS revealed translucent composition; Triton X-100 did not have the same effect on the muscle tissue and remnants of unaffected tissue in the final scaffold could be observed.

Standard haematoxylin eosin staining proved presumption of inefficient decellularization based on Triton X-100 due to presence of some nuclei. Skeletal muscle fibres with occasional presence of striation were observed and presence of several nuclei underneath the sarcolemma (Fig. 1C) could
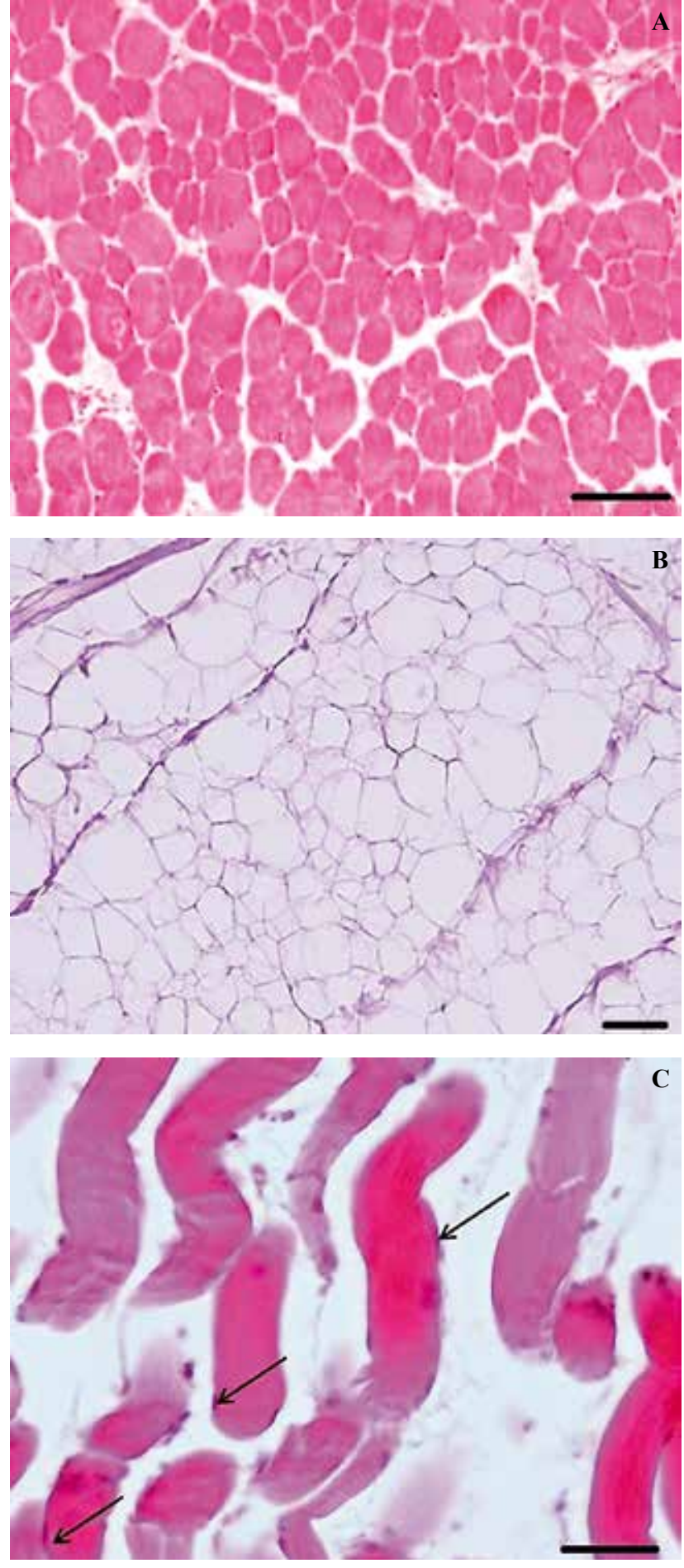

Fig. 1: Histological appearance after haematoxylin eosin staining of native muscle tissue (A) and muscle tissue treated with SDS (B) and Triton X-100 (C). Cross section of untreated muscle has typical architecture of skeletal muscle tissue while SDS-treated muscle shows disappearance of all cytoplasmic and nuclear components with preservation of ECM. Longitudinal section of a sample treated with Triton X-100 reveals presence of striated muscle fibres with preserved nuclei (arrows). Scale bar represents $50 \mu \mathrm{m}$. 
be detected. Decellularization was inefficient, because ECM components were disrupted and debris surrounded striated muscle fibres DAPI staining showed positive fluorescent signal because of nuclei presence (figure not shown).

\section{Sirius Red}

Collagen structure is highly desirable to preserve after decellularization mainly for its structural function to sustain a shape of the scaffold. Sirius staining proved preservation of collagen in scaffold treated by SDS without any loss of collagen density or visible disruption of collagen fibres (Fig. 2A). Figure 2B showed another example of ineffective decellularization with Triton X-100; collagen fibres were heavily damaged and presence of striated muscle fibres was observed. Deterioration of collagen fibres caused lack of cohesion in scaffold and resulted in deformation of scaffold.
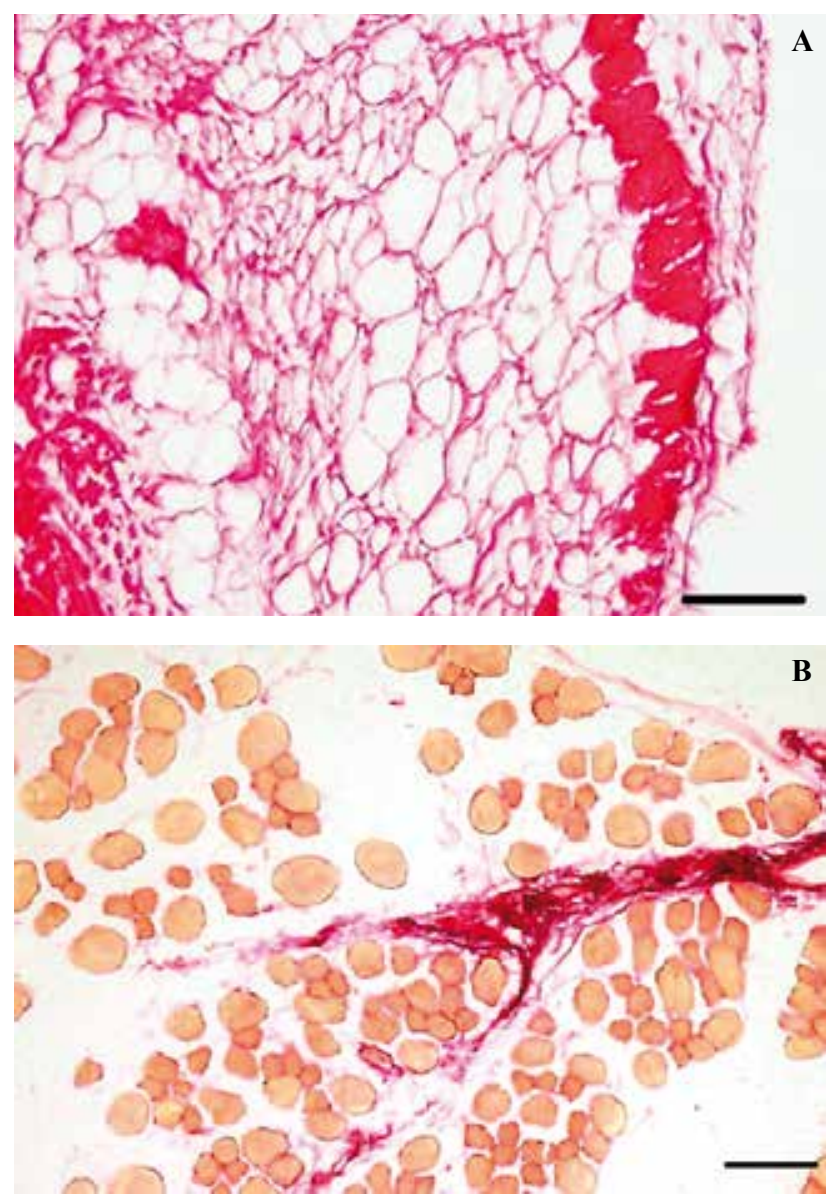

Fig. 2: Visualization of collagen fibres by Sirius Red staining in the skeletal muscle tissue decellularized by SDS (A) and Triton $\mathrm{X}-100$ (B). Collagen fibres are stained deeply red as they are major components of ECM; high density of red staining in case of SDS treated tissue was observed. Triton X-100 decellularization preserved collagen fibres as well, but they lacked intactness and they were significantly reduced. Scale bar represents $50 \mu \mathrm{m}$.

\section{Alcian blue staining}

Alcian blue is a polyvalent basic dye for visualization of glycosaminoglycans (GAG) as a part of the ground substance which plays an important role in water retention and growth factor adhesion. SDS treatment preserved GAGs (Fig. 3A), but light blue staining probably illustrated a partial loss of these macromolecules. Nevertheless, glycosaminoglycans were preserved and intact. Decellularization by Triton X-100 preserved GAGs as well and a density of blue staining showed higher amount of GAGs in samples treated with Triton X-100 than in scaffolds decellularized by SDS. Glycosaminoglycans, presented in Triton X-100 scaffold, showed disruption and fragmentation.
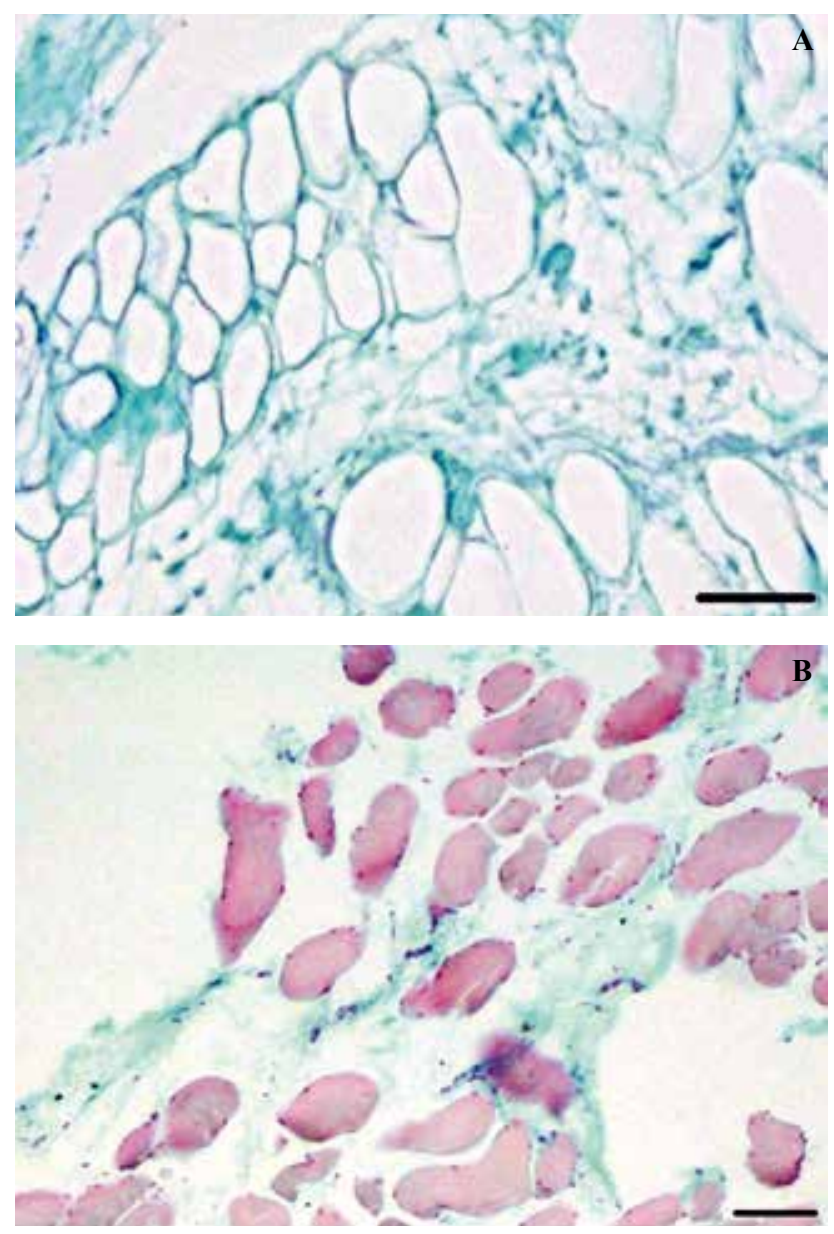

Fig. 3: Alcian blue staining illustrated presence of glycosaminoglycans in scaffold treated with SDS (A) or Triton X-100 (B). Scaffold prepared by SDS decellularization method demonstrates absence of striated muscle fibres and a typical honey-comb shape was preserved which is visualised by surrounding ECM, particularly blue coloured glycosaminoglycans. Triton treated samples also contained preserved glycosaminoglycans, but these scaffolds were unefficiently decellularized because of striated muscle fibres presence (pink stained structures with nuclei localized on the periphery, not shown in figure). Scale bar represents $50 \mu \mathrm{m}$. 


\section{Jones' impregnation method}

Preservation of the lamina externa was determined with impregnation technique by Jones. The lamina externa is an essential component for cell attachment and tissue regeneration so it is crucial to preserve this structure. The lamina externa contains silver-reactive sites which surround adipocytes, peripheral nerve fibres and first of all muscle fibres. Decellularization had no severe effect upon lamina externa structure and both samples preserved this structure stained in black colour. Figure 4 shows impregnation of basement membrane which belongs to muscle fibres or to blood capillaries.

\section{Resorcin-fuchsin staining}

Elastic fibres are arranged in a branching pattern to form a three dimensional network and they are localized closely to collagen fibres to limit their distensibility of the tissue
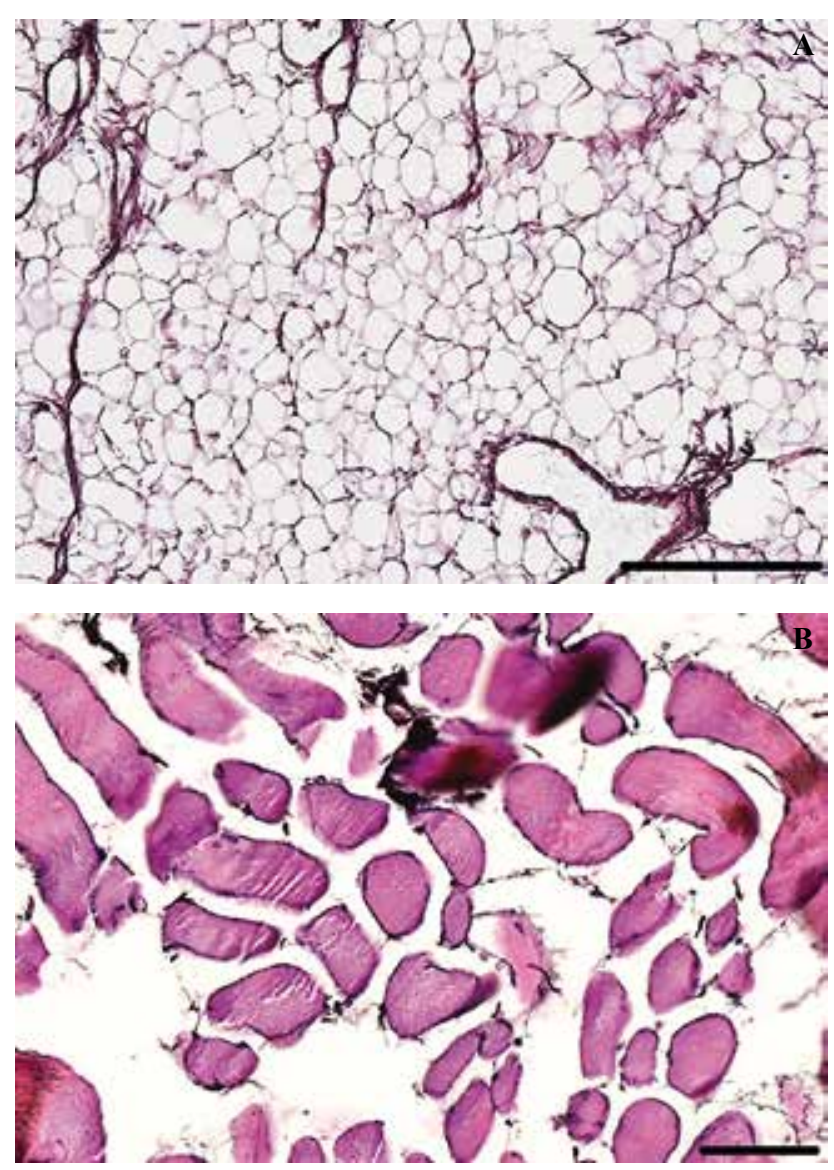

Fig. 4: Jones' impregnation revealed preservation of the basal lamina of decellularized samples processed by SDS (A) and Triton $\mathrm{X}-100$ (B). Basement membrane is black coloured and surrounds former muscle fibres in case of SDS decellularization and partially lysed muscle fibres in Triton X-100 sample. Scale bar represents $50 \mu \mathrm{m}$.
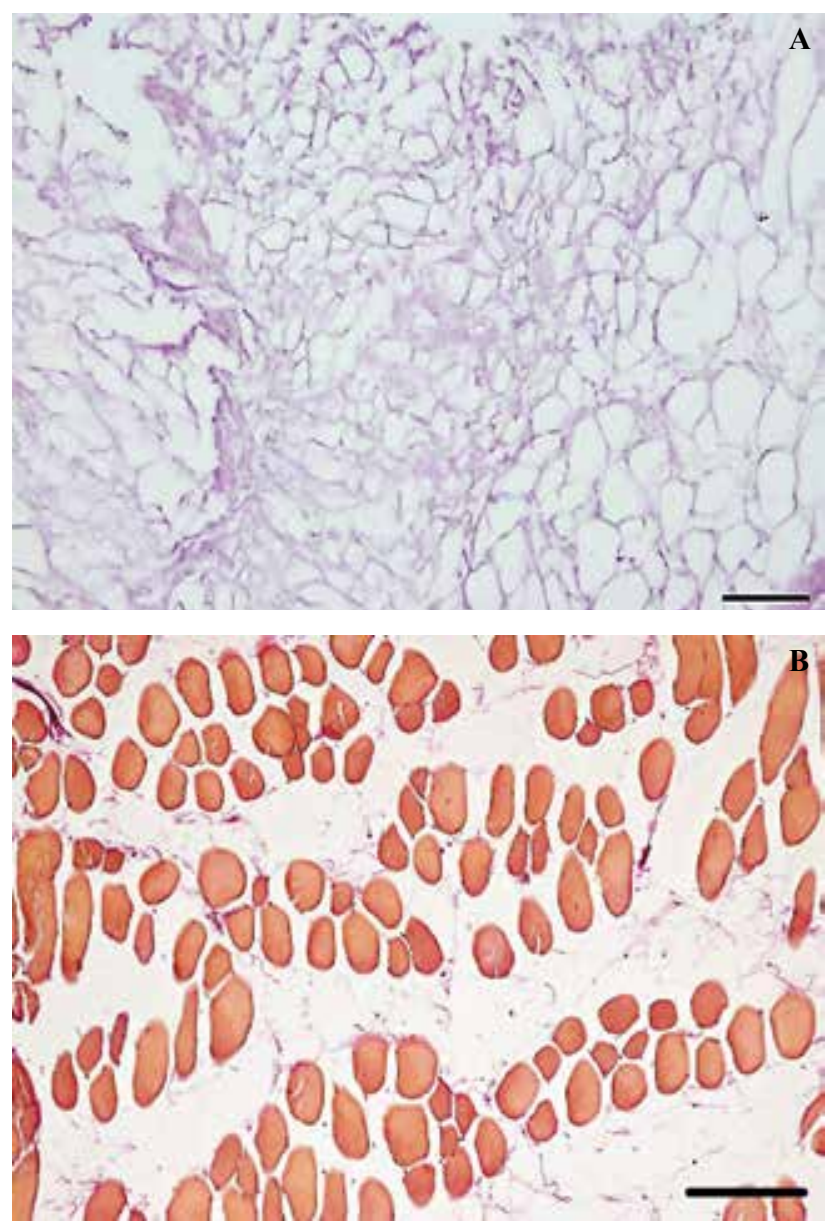

Fig. 5: Specific staining for elastic fibres, Resorcin-fuchsin, showed its preservation in samples decellularized by SDS (A) and Triton X-100 (B). Three dimensional network generated by elastic fibres was disrupted in case of Triton-decellularized samples which were visualised in violet colour, skeletal fibres by yellow to orange colour. Preserved nuclei were observed in Figure 5B and surrounding extracellular matrix changed according to its damage caused by Triton effect and a whole tissue had alternated microarchitecture. This architecture damage can be compared with the scaffold prepared by SDS decellularization (A) which preserved three dimensional network and all cytoplasmic and nuclear components were absent in this scaffold. Scale bar represents $50 \mu \mathrm{m}$.

and prevent tearing from excessive stretching. No distortion or fragmentation was observed in case of a scaffold treated by SDS agens (Fig. 5A); elastic fibres were stained slightly lighter than in case of Triton X-100 decellularization. Triton X-100 scaffold stained with resorcin-fuchsin showed disruption of elastic fibres (Fig. 5B).

\section{Discussion}

The main goal of this study was to clarify efficiency of two decellularization agents, SDS and Triton X-100, which 
are both the most used detergents to produce tissue or organ scaffolds. Effective decellularization process has minimal effect on the extracellular matrix, preserves a three dimensional microarchitecture of a tissue as a scaffold for tissue or organ reconstruction and most of all eliminates cellular material from the tissue. To verify effectiveness of individual decellularization agents, we analysed scaffolds with several histological techniques which evaluate ability of Triton $\mathrm{X}-100$ and SDS to eliminate cell nuclei and preserve main ECM components, such as collagen, glycosaminoglycans, basal lamina and elastic fibres.

Our idea to compare influence of Triton X-100 and SDS was based on studies (18-20) which used these two agents to prepare decellularized scaffolds. Both of these agents disrupt the cell membrane causing cell lysis by osmotic gradient. Detergents are effective in a cell removal from in the scaffold which can have a crucial role in an immune response, but they also can affect negatively structure of the scaffold; for instance, Triton X-100 can affect a decrease of GAG and damage ultrastructure of tissue or organ $(21,22)$. SDS also removes effectively cell nuclei and cytoplasmic components, especially from dense tissues, but they tend to damage collagen and decrease GAG content $(23,24)$, so they have very similar adverse consequence on the extracellular matrix. As both these agents are considered to be effective decellularization reagents, we would like to know which one is more effective in decellularizing the skeletal muscle tissue and for that reason we choose the same conditions in decellularization protocol (including length of decellularization, material and technique to apply the agent).

After decellularization, the skeletal muscle tissue appeared almost translucent in SDS protocol; Triton protocol did not affect a muscle so well and we could observe cytoplasmic debris in the tissue. Overall, our study proved higher efficiency of SDS agent to decellularize the skeletal muscle tissue which was supported by several histological findings. Haematoxylin eosin, as a standard method for the first tissue inspection, showed persistence of striated muscle fibres and nuclei after treatment with Triton X-100 which is according to criteria stated by Gilbert and Crapo (25) (lack of visible nuclear and cytoplasmic material in tissue section stained with haematoxylin and eosin staining) considered as ineffective decellularization. Elimination of cells from tissue or organ is crucial for subsequent implantation of the scaffold into living organism and significantly decreases a risk of rejection.

The extracellular matrix is a complex of structural and functional proteins and each type of tissue has specific composition of these proteins (26). The most abundant ECM protein is collagen which is present in several motifs as collagen type IV in basal membrane of vascular structures, collagen type I in collagen fibres, collagen type VII as an anchoring fibrils. Preservation of a different type of collagen was evaluated by two methods as Sirius Red method and Jones' impregnation. Sirius Red is specific staining method to distinguish collagen fibres, especially it allows determin- ing difference between collagen type I and III. This type of staining revealed no changes in collagen distribution after SDS cell extraction, either fragmentation or distortion. These results were not observed in Triton X-100 treatment; collagen fibres were unevenly distributed in ECM and were heavily damaged which could cause incoherence and mechanical endurance of tissue or organ. Triton protocol also decreased collagen density as reported in the study by Graus et al. (27). Elastin fibres accompany collagen fibres and are arranged in a branching pattern forming a three dimensional network. These fibres play an important role in distensibility of the tissue and can prevent tearing from stretching. SDS and Triton X-100 treatment of the skeletal muscle tissue preserved elastin fibres, but Triton X-100 damaged fibres morphology and altered molecular structure of elastin resulting in uneven distribution of elastic fibres. SDS decellularization affects elastin fibres in content which was decreased. Laminin, collagen type IV and anchoring fibrils are the most prominent structures in basement membrane and they specify its ultrastructure and function as an attachment site for cells, filtration, and regulation and also provide scaffold during regeneration (28). Decellularized samples processed from both decellularized agents revealed intactness of basement membrane as visualized by Jones' impregnation.

The ability of preserving glycosaminoglycans was also compared in both skeletal muscle tissues treated by SDS and Triton X-100. SDS decellularization protocol successfully retains these heteropolysaccharides which are bound to proteoglycans and serve as binding sites for growth factors, cytokines and contribute to water retention due to their negative charge (29). Triton X-100 treatment results in significant elimination of GAGs from the skeletal muscle tissue as analysed by Alcian blue staining. Reduced content of GAGs in the scaffold may decrease mechanical resistance because of GAGs are responsible for gel consistency which can resist deformations during tissue function $(24,28)$. Decellularization with SDS as a main agent was more successful in term of GAGs preservation, but amount of these macromolecules was also decreased. These results are consistent with other studies $(30,21)$ using SDS as a main decellularization agent.

\section{Conclusion}

ECM alteration or damage can be caused during decellularization by several factors including type of used detergent, concentration of detergent, duration of decellularization process, presence of protease inhibitor etc. (31). Differences between various decellularization agents are based upon their function and these variations determine discrepancies in effect on the extracellular matrix. Our study proves that ionic detergent SDS is sufficient agent for cells removal in the skeletal muscle tissue and preservation of the extracellular matrix microarchitecture providing microenvironment suitable for cell attachment and growth. 


\section{Acknowledgements}

This work was supported by the Grant Agency of the Czech Republic 15-09161S, Grant of the Charles University No. SVV-2016-260278 and PRVOUK P37/06.

\section{Abbreviation}

ECM extracellular matrix

DAPI 4',6-diamidino-2-phenylindole

GAG glycosaminoglycans

PBS phosphate buffered saline

SDS sodium dodecyl sulphate

\section{References}

1. Cheng CW, Solorio LD, Alsberg E. Decellularized tissue and cell-derived extracellular matrices as scaffolds for orthopaedic tissue engineering. Biotechnol Adv 2014; 32: 462-84

2. Hurd SA, Bhatti NM, Walker AM, Kasukonis BM, Wolchok JC. Development of a biological scaffold engineered using the extracellular matrix secreted by skeletal muscle cells. Biomaterials 2015; 49: 9-17.

3. Wang L, Johnson JA, Chang DW, Zhang Q. Decellularized musculofascial extracellular matrix for tissue engineering. Biomaterials 2013; 34: 2641-54.

4. Criswell TL, Corona BT, Wang $Z$ et al. The role of endothelial cells in myofiber differentiation and the vascularization and innervation of bioengineered muscle tissue in vivo. Biomaterials 2013; 34: 140-9.

5. Yamamoto K, Murphy G, Troeberg L. Extracellular regulation of metalloproteinases. Matrix Biol 2015; 44-46: 255-63.

6. Schultz GS, Wysocki A. Interactions between extracellular matrix and growth factors in wound healing. Wound Repair Regen 2009; 17: 153-62.

7. Reddy PP, Barrieras DJ, Wilson G et al. Regeneration of functional bladder substitutes using large segment acellular matrix allografts in a porcine model. J Urol 2000; 164: 936-41.

8. Rosario DJ, Reilly GC, Salah EA, Glover M, Bullock AJ, MacNeil S. Decellularization and sterilization of porcine urinary bladder matrix for tissue engineering in the lower urinary tract. Regen Med 2008; 3: 145-56.

9. Quarti A, Nardone S, Colaneri M, Santoro G, Pozzi M. Preliminary experience in the use of an extracellular matrix to repair congenital heart diseases. Interact Cardivasc Thorac Surg 2011; 6: 659-72.

10. Dong J, Li Y, Mo X. The study of a new detergent (octyl-glucopyranoside) for decellularizing porcine pericardium as tissue engineering scaffold. J Surg Res 2013; 183: 56-67.

11. Weber B, Dijkman PE, Scherman J et al. Off-the-shelf human decellularized tissueengineered heart valves in a non-human primate model. Biomaterials 2013; 34 7269-80

12. Nonaka PN, Uriarte JJ, Campillo N et al. Mechanical properties of mouse lungs along organ decellularization by sodium dodecyl sulfate.Respir Physiol Neurobiol 2014; $200: 1-5$.
13. O'Neill JD, Anfang R, Anandappa A et al. Decellularization of human and porcine lung tissues for pulmonary tissue engineering. Ann Thorac Surg 2013; 96 : 1046-55.

14. Gupta SK, Dinda AK, Potdar PD, Mishra NC. Fabrication and characterization of scaffold from cadaver goat-lung tissue for skin tissue engineering applications. Mater Sci Eng C Mater Biol Appl 2013; 33: 4032-8.

15. Su Z, Ma H, Wu Z et al. Enhancement of skin wound healing with decellularized scaffolds loaded with hyaluronic acid and epidermal growth factor. Mater Sci Eng C Mater Biol Appl 2014; 44: 440-8

16. Baptista MP, Siddiqui MM, Lozier G, Rodriguez SR, Atala A, Soker S. The use of whole organ decellularization for the generation of a vascularized liver organoid. Hepatology 2011; 53: 604-17.

17. Ott $\mathrm{CH}$, Mathiesen TS, Goh SK et al. Perfusion-decellularized matrix: using nature's platform to engineer a bioartificial heart. Nature Medicine 2007; 14: 213-21.

18. Ungerleider JL, Johnson TD, Rao N, Christman KL. Fabrication and characterization of injectable hydrogels derived from decellularized skeletal and cardiac muscle. Methods 2015; 84: 53-9.

19. Perniconi B, Costa A, Aulino P, Teodori L, Adamo S, Coletti D. The pro-myogenic environment provided by whole organ scale acellular scaffolds from skeletal muscle. Biomaterials 2011; 32: 7870-82.

20. Caralt M, Uzarski JS, Iacob S et al. Optimization and critical evaluation of decellularization strategies to develop renal extracellular matrix scaffolds as biological templates for organ engineering and transplantation. Am J Transplant 2015; 15 64-75.

21. Ott HC, Matthiesen TS, Goh SK et al. Perfusion decellularized matrix: using nature's platform to engineer a bioartificial heart. Nat Med 2008; 14: 213-21.

22. Uygun BE, Soto-Gutierrez A, Yagi H et al. Organ reengineering through development of a transplantable recellularized liver graft using decellularized liver matrix. Nat Med 2010; 16: 814-20.

23. Reing JE, Brown BN, Daly KA et al. The effects of processing methods upon mechanical and biologic properties of porcine dermal extracellular matrix scaffolds. Biomaterials 2010; 31: 8626-33.

24. Du L, Wu X, Pang K, Yang Y. Histological evaluation and biomechanical characterisation of an acellular porcine cornea scaffold. Br J Ophthalmol 2011; 95: 410-4.

25. Crapo PM, Gilbert TW, Badylak SF. An overview of tissue and whole organ decellularization processes. Biomaterials 2011; 32: 3233-43.

26. Hrebikova H, Diaz D, Mokry J. Chemical decellularization: a promising approach for preparation of extracellular matrix. Biomed Pap Med Fac Univ Palacky Olomouc Czech Repub 2015; 159: 12-7.

27. Grauss RW, Hazekamp MG, Oppenhuizen F, Munsteren CJ, Gittenberger-de Groot AC, DeRuiter MC. Histological evaluation of decellularized porcine aortic valves: matrix changes due to different decellularization methods. Eur J Cardiothorac Surg 2005; 27: 566-71.

28. Ross MH, Pawlina W. Histology, text and atlas: with correlated cell and molecular biology. 6th ed. Philadelphia: Wolter Kluwer, 2011.

29. Badylak SF. Xenogeneic extracellular matrix as a scaffold for tissue reconstruction. Transpl Immunol 2004; 12: 367-77.

30. Gilpin SE, Guyette JP, Gonzalez G et al. Perfusion decellularization of human and porcine lungs: bringing the matrix to clinical scale. J Heart Lung Transplant 2014; 33: $298-308$.

31. Gilbert TW, Sellaro TL, Badylak SF. Decellularization of tissues and organs. Biomaterials $2006 ; 27: 3675-83$.

Received: 03/08/2016

Accepted: 19/10/2016 